\title{
Prevalencia de Eimeria spp en Alpacas de Dos Comunidades del Distrito de Macusani, Puno, Perú
}

\author{
Prevalence of Eimeria spp in Alpacas of Two Communities in the Macusani \\ District, Puno, Peru
}

\author{
Elizabeth Camareno H. ${ }^{1}$, Amanda Chávez V. ${ }^{1,3,4}$, Rosa Pinedo V. ${ }^{1}$, Víctor Leyva V. ${ }^{2}$
}

\section{Resumen}

\begin{abstract}
El estudio tuvo por objetivo determinar la prevalencia de Eimeria spp en alpacas de dos comunidades del distrito de Macusani, provincia de Carabaya, Puno, durante la época seca, según procedencia, estrato etario ( 5 meses a $<1$ año, 1-3 años, $>3$ años) y sexo. Se colectaron 1319 muestras coprológicas de alpacas Huacaya, siendo 598 de la comunidad Hatun Phinaya y 721 de la comunidad Queracucho, entre agosto y octubre de 2010. Las muestras se evaluaron mediante las técnicas cualitativas de sedimentación y flotación en soluciones de Willis y Sheather. La cuantificación de la carga parasitaria se hizo mediante el método de McMaster modificado y para identificar las especies de eimerias se realizó la esporulación y medición de ooquistes. Se encontró una alta prevalencia de eimerias $(52.4 \pm 2.7 \%)$. El análisis de riesgo mediante regresión logística múltiple demostró que las alpacas de 5 meses a $<1$ año y aquellas de 1 a 3 años tuvieron 13.2 y 2.4 veces, respectivamente, más riesgo de infección que las $>3$ años $(\mathrm{p}<0.05)$. Se encontraron cinco especies de eimerias, con una frecuencia para las grandes eimerias de $8.7 \%$ de E. macusaniensis y $0.7 \%$ de E. ivitaensis. Entre las pequeñas eimerias (E. punoensis, E. alpacae y E. lamae), E. punoensis presentó la mayor frecuencia (66.2\%). La carga parasitaria de eimerias fue baja (187.8 opg). Los resultados confirman que la eimeriosis constituye un problema potencial de salud en las alpacas de las dos comunidades alpaqueras del distrito de Macusani, Puno.
\end{abstract}

Palabras clave: alpacas, Eimeria, prevalencia, Macusani, época seca

\footnotetext{
${ }^{1}$ Laboratorio de Microbiología y Parasitología Veterinaria, ${ }^{2}$ Laboratorio de Reproducción Animal, Facultad de Medicina Veterinaria, Universidad Nacional Mayor de San Marcos, Lima, Perú

${ }^{3}$ Estudio financiado por PROVIAS, Perú

${ }^{4}$ E-mail: achavezvg@gmail.com
}

Recibido: 18 de setiembre de 2015

Aceptado para publicación: 25 de abril de 2016 
The aim of this study was to determine the prevalence of Eimeria spp in alpacas of two communities of the Macusani district, Carabaya province, Puno, Peru, during the dry season, accodring to origin, age ( 5 month to $<1$ year, 1-3 years, $>3$ years) and sex. Coprological samples $(\mathrm{n}=1319)$ were collected from Huacaya alpacas $(598$ from the Hatun Phinaya community and 721 from the Queracucho community) during August and October 2010. Samples were analyzed by qualitative techniques (sedimentation and flotation using Sheather and Willis solutions). The quantification of Eimeria load was done using the modified McMaster method and the oocyst sporulation and measurement was done to identify the species of eimerias. The results showed a prevalence of $52.4 \%$ for Eimeria spp. Risk analysis by multiple logistic regresion test showed alpacas of 5 months to $<1$ year and those of 1 to 3 years had 13.2 and 2.4 times higher risk to become infected to Eimeria sp than those over 3 years $(\mathrm{p}<0.05)$. Five Eimeria species were identified, where the frequency of large eimerias was $8.7 \%$ for $E$. macusaniensis and $0.7 \%$ for $E$. ivitaensis. Among the small Eimeria species (E. punoensis, E. alpacae, E. lamae), E. punoensis showed the greatest frequency (66.2\%). The parasitic load of eimeria was considered low (187.8 opg). These findings confirm that eimeriosis is a potential healthy problem in alpacas of the two Macusani communities.

Key words: alpaca, Eimeria, prevalence, Macusani, dry season

\section{INTRODUCCIÓN}

A nivel nacional, Puno constituye el primer departamento con mayor población de alpacas y de producción de fibra de alpaca. Las comunidades y parcelas campesinas poseen el $80-85 \%$ de estos animales (INEI, 2010). Asimismo, el distrito de Macusani, capital de la provincia de Carabaya, Puno, se le considera la capital alpaquera del Perú y del mundo (Ocola, 2008), ya que el $40.9 \%$ de su población económicamente activa se encuentra dedicada a la crianza de camélidos sudamericanos y a la agricultura en general (INEI, 2007). No obstante, la producción de carne y fibra se encuentra limitada por enfermedades parasitarias, bacterianas y virales, siendo los problemas parasitarios los más frecuentes (Mamani et al., 2009).

Las eimerias son protozoos específicos e intracelulares que presentan tres fases reproductivas dentro de su ciclo biológico (esquizogonia, gametogonia y esporogonia) (Leguía y Casas, 1999). El huésped se infec- ta por vía oral al ingerir las formas infectivas (ooquistes esporulados), los cuales liberan los esporozoitos al llegar al intestino delgado. Al alcanzar la madurez sexual inician la liberación de ooquistes no esporulados que salen al medio ambiente con las heces, logrando esporular entre 8 y 33 días, dependiendo de las condiciones ambientales y de la especie de eimeria (Leguía y Casas, 1999). En los camélidos sudamericanos se han descrito seis especies, siendo estas Eimeria punoensis, E. alpacae, E. lamae, E. peruviana, E. macusaniensis y E. ivitaensis (Leguía y Casas, 1999).

La eimeriosis produce una infección intestinal, cuya patogenicidad depende del tipo de eimeria. La asociación de especies más patógena es E. macusaniensis con E. lamae (Palacios et al., 2004), la que ocasiona inflamación de la mucosa intestinal, abundante mucosidad y epitelio descamado con presencia de sangre, afectando la absorción de nutrientes $\mathrm{y}$, por tanto, el rendimiento productivo de las alpacas (Ramírez et al., 1998). La presentación subclínica es la más común en 
adultos, en tanto que en crías se presenta mayormente en forma clínica (Fowler, 1998), pudiendo causar mortalidad (Rosadio y Ameghino, 1994; Palacios et al., 2006; Rosadio et al., 2012). Por otro lado, las madres y los machos que ingresan a la majada para el empadre podrían ser portadores sanos de la enfermedad y fuente de infección, incrementando la contaminación de las canchas de pastoreo (Martín et al., 2010).

El diagnóstico de eimeriosis se realiza con la observación de los ooquistes en heces, así como mediante la identificación de lesiones histopatológicas patognomónicas de la enfermedad en el intestino delgado y grueso (Leguía y Casas, 1999).

Se reportan prevalencias de eimeriosis en alpacas, llamas y vicuñas de 30 a 100\% (Leguía y Casas, 1999). La prevalencia puede llegar al 90\% a los 4-5 meses de edad, para luego descender a niveles bajos, donde las alpacas se convierten en portadores del parásito (Rojas, 2004). Por otro lado, es posible que muchas de las crías registradas como muertas por 'diarrea común', enterotoxemia o colibacilosis podrían corresponder a cuadros de eimeriosis (Ameghino y De Martini, 1991).

La gran mayoría de estudios sobre eimeriosis han sido realizados en crías de alpacas, siendo necesario conocer la situación actual de esta parasitosis en los diversos grupos etarios. Por ello, el objetivo del estudio fue estimar la prevalencia, carga y especies de eimerias en alpacas de dos comunidades del distrito de Macusani, Puno, así como determinar posibles factores de riesgo.

\section{Materiales Y MÉTOdos}

\section{Lugar de Estudio}

El estudio se realizó en las comunidades de Hatun Phinaya y Queracucho, en el distrito de Macusani, provincia de Carabaya, departamento de Puno, Perú, entre agosto y octubre de 2010. El distrito tiene una extensión de $1030 \mathrm{~km}^{2}$ (INEI, 2007) y está situado entre 4315 a $4480 \mathrm{msnm}$. Presenta una precipitación pluvial anual de 400 a $700 \mathrm{~mm}$, temperatura media anual de $5{ }^{\circ} \mathrm{C}$, con máximas de $13.3{ }^{\circ} \mathrm{C}$ y mínimas de $-7.2^{\circ} \mathrm{C}$, y humedad anual de $76 \%$. Su topografía comprende colinas, mesetas y cumbres andinas. La época de seca se circunscribe a los meses de abril a noviembre y los demás meses corresponden a la época de lluvias (SENAMHI, 2010).

\section{Tamaño Muestral}

El número mínimo de animales fue determinado mediante la fórmula para estimar proporción de poblaciones finitas (Daniel, 2002), contando con el apoyo voluntario de los comuneros residentes de la zona de estudio y con el soporte de PROVÍAS, dentro del Proyecto «Mejoramiento genético de las alpacas tramo II y III». Para el estudio, se consideró una población de 4692 alpacas en las dos comunidades y se utilizó una prevalencia de 61\%, hallada por Wolf (2010), dando como resultado un tamaño de muestra mínima de 340 alpacas. Sin embargo, en el estudio se muestrearon 1319 alpacas.

\section{Recolección y Análisis de Muestras}

Se obtuvieron entre 40 y $50 \mathrm{~g}$ de heces por animal, directamente del recto, identificándose la procedencia (Hatun Phinaya, Queracucho), estrato etario (5 meses a $<1$ año; 1 a 3 años; $>3$ años) y sexo. Las muestras fueron almacenadas en recipientes térmicos con refrigerante para su traslado al Laboratorio de Parasitología Veterinaria de la Facultad de Medicina Veterinaria de la Universidad Nacional Mayor de San Marcos, en Lima.

En la evaluación coproparasitológica se utilizaron técnicas cualitativas de sedimentación espontánea, flotación con solución de Willis y la técnica cuantitativa de McMaster modificado (Rojas, 2004). Mediante la técnica de sedimentación espontánea se determi- 
nó la prevalencia de E. macusaniensis y $E$. ivitaensis. En el caso de muestras positivas a pequeñas eimerias (E. punoensis, E. alpacae y E. lamae), se les realizó la esporulación y se midieron los ooquistes para su identificación siguiendo las referencias de Guerrero et al. (1970b) y Leguía y Casas (1999).

\section{Análisis Estadístico}

Se calculó la prevalencia de eimerias mediante la determinación del número de muestras fecales positivas con un intervalo de confianza de $95 \%$. Las variables procedencia, estrato etario y sexo fueron analizadas por el odds ratio mediante regresión logística (Daniel, 2002). La posible asociación entre estas variables y la presencia de las especies de eimerias se determinó mediante la prueba de Chi Cuadrado. Los datos se procesaron utilizando el paquete estadístico SPSS v. 20.0 .

Se calcularon las medias genométricas de las cargas parasitaria de eimerias, estableciéndose el grado de infección en alpacas con base en reportes hallados en rumiantes por Radostits et al. (1999).

\section{Resultados}

La prevalencia general de eimerias en alpacas de las dos comunidades de Macusani, Puno, fue de $52.4 \pm 2.7 \%$ (Cuadro 1). Las variables procedencia y sexo no constituyeron factores de riesgo para la infección por eimerias; sin embargo, las alpacas de 5 meses a un año de edad y de 1 a 3 años de edad mostraron un riesgo de 13.2 y 2.4 veces mayor respecto al estrato basal ( $>3$ años), ajustado a la variable procedencia y sexo (Cuadro 1).

La media geométrica fue de 187.8 ooquistes por gramo de heces (opg). En forma similar, el $89 \%$ de los animales presentaron cargas $\leq 1000$ opg y solo el $2 \%$ eliminó cargas altas, mayores de 5000 opg, alcanzando un máximo de 82400 opg (Cuadro 2).

En el Cuadro 3 se presenta la prevalencia de eimerias por especies. Se encontraron diferencias significativas $(\mathrm{p}<0.05)$ según estrato etario, tanto para E. macusaniensis como para las pequeñas eimerias $(E$. punoensis, E. alpacae y E. lamae), observándose que alpacas de 5 meses a menos de 1 año presentaron una mayor prevalencia en comparación con los demás grupos etarios.

Al realizar la esporulación de los ooquistes para diferenciar las tres pequeñas eimerias, se pudo determinar un mayor porcentaje de E. punoensis (66.2\%), a diferencia de E. alpacae y E. lamae, quienes presentaron frecuencias de 31.5 y $2.3 \%$, respectivamente. Asimismo, se evidenció la predominancia de E. lamae (5.1\%) en alpacas de 5 meses a menos de 1 año con respecto a los otros grupos etarios.

\section{Discusión}

La elevada prevalencia de Eimeria spp (52.4\%) en las alpacas de las comunidades evaluadas fue similar a reportes históricos de prevalencias encontradas en zonas aledañas: La Raya (58.1\%) y Quinsachata (61.3\%) (Guerrero et al., 1970b; Wolf, 2010). Este resultado evidencia la existencia permanente de algunas condiciones en la zona de estudio que favorecerían la presencia de eimerias en alpacas. Así, características climatológicas (humedad, temperatura, tiempo de radiación solar), topografía que comprende colinas, mesetas y cumbres andinas cubiertas por champas de pasto natural (ichu), yaretas y cactáceas, junto con la presencia de riachuelos provenientes del deshielo de los nevados favorecen un microclima favorable para el desarrollo de los ooquistes. Los meses más húmedos y templados (diciembre a marzo) son la época ideal para la esporulación de los ooquistes y el mantenimiento de las formas infectivas, y que coincide con la época de 
Cuadro 1. Prevalencia de Eimeria spp en alpacas Huacaya de dos comunidades del distrito de Macusani, Puno (agosto - octubre, 2010) y variables asociadas

\begin{tabular}{lcccccc}
\hline & $\begin{array}{c}\text { Alpacas } \\
\left.\text { (N. }{ }^{\circ}\right)\end{array}$ & \multicolumn{2}{c}{ Positivos } & \multicolumn{3}{c}{ Regresión logística múltiple } \\
\cline { 3 - 7 } & & Número & $\% \pm \mathrm{IC}^{1}$ & $\mathrm{OR}^{2}$ & $\mathrm{P}^{3}$ & IC \\
\hline $\begin{array}{l}\text { Procedencia } \\
\text { (comunidad) }\end{array}$ & & & & & & \\
$\quad$ Hatun Phinaya & 598 & 325 & $54.3 \pm 4.0$ & 1.28 & $>0.05$ & $1.0-1.6$ \\
$\quad \begin{array}{l}\text { Queracucho } \\
\text { Estrato etario }\end{array}$ & 721 & 366 & $50.8 \pm 3.6$ & 1 & - & - \\
$\quad 5 \mathrm{~m}$ a $<1$ año & 310 & 265 & $85.5 \pm 3.9$ & 13.21 & $<0.01$ & $9.0-19.4$ \\
1 a 3 años & 520 & 268 & $51.5 \pm 4.3$ & 2.35 & $<0.01$ & $1.8-3.1$ \\
$>3$ años & 489 & 158 & $32.3 \pm 4.1$ & 1 & - & - \\
Sexo & & & & & & \\
$\quad$ Hembra & 1051 & 529 & $50.3 \pm 3.0$ & 1.17 & $>0.05$ & $0.9-1.6$ \\
$\quad$ Macho & 268 & 162 & $60.4 \pm 5.9$ & 1 & - & - \\
\hline Total & 1319 & 691 & $52.4 \pm 2.7$ & & & \\
\hline${ }^{1}$ Intervalo de confianza; ${ }^{2}$ Odds ratio; ${ }^{3}$ Nivel de significancia estadística & &
\end{tabular}

Cuadro 2. Media geométrica y valor máximo de ooquistes por gramo de heces (opg) de eimerias en alpacas Huacaya $(n=1319)$ de dos comunidades del distrito de Macusani, Puno (agosto-octubre, 2010)

\begin{tabular}{|c|c|c|c|c|}
\hline & & $\begin{array}{c}\text { Muestras } \\
\text { positivas } \\
\text { (n) }\end{array}$ & $\begin{array}{l}\text { Media } \\
\text { geométrica } \\
\text { (opg) }\end{array}$ & $\begin{array}{c}\text { Carga máxima } \\
\text { (opg) }\end{array}$ \\
\hline \multicolumn{5}{|c|}{$\begin{array}{l}\text { Procedencia } \\
\text { (comunidad) }\end{array}$} \\
\hline & Hatun Phinaya & 325 & 208.3 & 66,150 \\
\hline & Queracucho & 366 & 171.3 & 82,400 \\
\hline \multicolumn{5}{|c|}{ Estrato etario } \\
\hline & $5 \mathrm{~m} \mathrm{a}<1$ año & 265 & 401.6 & 82,400 \\
\hline & 1 a 3 años & 268 & 129.4 & 5,050 \\
\hline & $>3$ años & 158 & 98.7 & 5,400 \\
\hline \multicolumn{5}{|l|}{ Sexo } \\
\hline & Hembras & 529 & 173.7 & 82,400 \\
\hline & Machos & 162 & 242.4 & 38,100 \\
\hline Total & & 691 & 187.8 & 82,400 \\
\hline
\end{tabular}


Cuadro 3. Prevalencia de las grandes y pequeñas eimerias en alpacas Huacaya de dos comunidades del distrito de Macusani, Puno (agosto - octubre, 2010)

\begin{tabular}{|c|c|c|c|c|c|c|c|}
\hline & \multirow{3}{*}{$\begin{array}{c}\text { Total } \\
\text { (n) }\end{array}$} & \multicolumn{4}{|c|}{ Grandes eimerias } & \multirow{2}{*}{\multicolumn{2}{|c|}{ Pequeñas eimerias ${ }^{1}$}} \\
\hline & & \multicolumn{2}{|c|}{ E. macusaniensis } & \multicolumn{2}{|c|}{ E. ivitaensis } & & \\
\hline & & $\begin{array}{l}\text { Positivas } \\
\text { (n) }\end{array}$ & $\% \pm \mathrm{IC}$ & $\begin{array}{l}\text { Positivas } \\
\text { (n) }\end{array}$ & $\% \pm \mathrm{IC}$ & $\begin{array}{l}\text { Positivas } \\
\text { (n) }\end{array}$ & $\% \pm \mathrm{IC}$ \\
\hline \multicolumn{8}{|l|}{$\begin{array}{l}\text { Procedencia } \\
\text { (comunidad) }\end{array}$} \\
\hline $\begin{array}{l}\text { Hatun } \\
\text { Phinaya }\end{array}$ & 598 & 59 & $9.9 \pm 2.4$ & 5 & $0.8 \pm 0.8$ & 323 & $54.0 \pm 4.0$ \\
\hline Queracucho & 721 & 56 & $7.8 \pm 2.0$ & 4 & $0.6 \pm 0.7$ & 366 & $50.8 \pm 3.6$ \\
\hline \multicolumn{8}{|l|}{ Estrato etario } \\
\hline $5 \mathrm{~m} \mathrm{a}<1$ año & 310 & 90 & $29.0 \pm 5.1^{\mathrm{a}}$ & 4 & $1.3 \pm 1.1$ & 264 & $85.2 \pm 4.0$ \\
\hline 1 a 3 años & 520 & 15 & $2.9 \pm 1.5^{\mathrm{b}}$ & 4 & $0.8 \pm 0.9$ & 268 & $51.5 \pm 4.3^{t}$ \\
\hline$>3$ años & 489 & 10 & $2.0 \pm 1.2^{\mathrm{c}}$ & 1 & $0.2 \pm 0.4$ & 157 & $32.1 \pm 4.1$ \\
\hline \multicolumn{8}{|l|}{ Sexo } \\
\hline Hembra & 1051 & 78 & $7.4 \pm 1.5^{\mathrm{a}}$ & 7 & $0.7 \pm 0.6$ & 528 & $50.2 \pm 3.0$ \\
\hline Macho & 268 & 37 & $13.8 \pm 4.2^{\mathrm{b}}$ & 2 & $0.7 \pm 1.2$ & 161 & $60.1 \pm 5.9^{1}$ \\
\hline Total & 1319 & 115 & $8.7 \pm 1.5$ & 9 & $0.7 \pm 0.5$ & 689 & $52.1 \pm 2.7$ \\
\hline
\end{tabular}

parición de las alpacas en el altiplano, haciéndolas más susceptibles de infección (Romero, 1992; Leguía y Casas, 1999).

El estrato etario constituyó un factor de riesgo para la presentación de Eimeria spp (Cuadro 1), donde las alpacas más jóvenes (menores de un año de edad) presentaron 13.2 veces mayor susceptibilidad a la infección parasitaria que animales mayores de 3 años. Prevalencias similares fueron reportadas en crías menores de 3 meses de edad en Junín (90\%) por Romero (1992) y en Puno (87.5\%) por Rodríguez et al. (2012). Estos resultados reafirman la mayor susceptibilidad de los animales más jóvenes y la adquisición de resistencia conforme se incrementa la edad (Rojas, 2004). Por otro lado, la ausencia de diferencias estadísticas según procedencia y sexo (Cuadro 1) podría deberse a que los anima- les estuvieron en similares condiciones de pastoreo, quedando expuestos a los mismos riesgos de infección.

Aunque las prevalencias de Eimeria spp en ambas comunidades fueron elevadas, 'cargas bajas' de ooquistes (d» 1000 opg) se presentaron en el $89 \%$ de los animales positivos a eimerias, con un promedio general de 187.8 opg (Cuadro 3), lo cual no es indicativo de infección (Radostits et al., 1999). La costumbre que tienen las alpacas de defecar en sitios específicos impediría la diseminación del parásito de manera uniforme en las pasturas, evitando infecciones mayores. No obstante, la presencia de lluvias en la zona proporcionaría un microclima húmedo favorable para el desarrollo y viabilidad de los ooquistes en las letrinas (Leguía y Casas, 1999). 
Al evaluar la prevalencia hallada en las grandes y pequeñas eimerias por estrato etario, se encontró diferencia significativa $(\mathrm{p}<0.05)$, siendo la prevalencia de $E$. macusaniensis $(29 \%)$ en alpacas de 5 meses a $<1$ año mayor que en los otros estratos etarios (Cuadro 3). De igual manera, Guerrero et al. (1970) evidenciaron mayor prevalencia de E. macusaniensis en alpacas menores de 1 año (77.5\%) que en las mayores de un año (17.5\%), en tanto que Rodríguez et al. (2012) hallaron una alta prevalencia $(50 \%)$ en crías menores de 3 meses. Estos hallazgos evidencian el riesgo potencial de esta eimeria, especialmente debido al daño que produce en las criptas intestinales, la cual podría dejar expuesta la mucosa intestinal a otros agentes del complejo diarreico neonatal de las alpacas (Clostridium perfringens, Cryptosporidium spp, E. coli enterotoxigénica, etc.) (Martín et al., 2010; Rosadio et al., 2010).

El mayor porcentaje de infección por pequeñas eimerias correspondió a $E$. punoensis, lo cual se debería a múltiples factores externos (condiciones climáticas y ecológicas propicias). Asimismo, $E$. punoensis posee un menor tiempo de esporulación ( 8.5 a 12 días) y menor periodo prepatente ( 9 días) en comparación con las otras eimerias (Leguía y Casas, 1999). Por otro lado, cabe indicar que estudios experimentales han demostrado que infecciones con E. alpacae y E. punoensis no producen sintomatología clínica en alpacas mayores de un año (Chigerwe et al., 2007).

Las mayores predominancias de $E$. lamae (5.1\%) y E. macusaniensis $(29.0 \%)$ en alpacas menores de 1 año son un claro indicativo que muchos animales podrían estar infectados con estas dos eimerias, cuya asociación es altamente patógena (Palacios et al., 2004). E. lamae destruye el epitelio intestinal y E. macusaniensis causa atrofia y necrosis de las glándulas crípticas, ocasionando una diarrea hemorrágica que conduce a deshidratación, anemia, cólico, inapetencia $\mathrm{y}$, por último, la muerte del animal (Leguía y Casas, 1999).

\section{Conclusiones}

- Se encontró una alta prevalencia de Eimeria spp $(52.4 \pm 2.7 \%)$ en alpacas de dos comunidades del distrito de Macusani, Puno.

- Las alpacas entre 5 meses y 1 año de edad y de 1 a 3 años de edad mostraron 13.2 y 2.4 veces mayor riesgo de presentación de Eimeria spp, respectivamente, que alpacas mayores de 3 años.

- La carga parasitaria promedio fue baja (187.8 opg), donde solo el $2 \%$ de las alpacas presentó recuentos superiores a 5000 opg.

- Las especies de eimeria encontradas fueron E. punoensis, E. alpacae, E. lamae, E. macusaniensis y E. ivitaensis.

\section{Literatura Citada}

1. Ameghino E, DeMartini J. 1991. Coccidiosis. En: Mortalidad en crías de alpaca. Bol Div IVITA(Perú). p 71-112.

2. Daniel W. 2002. Bioestadística: bases para el análisis de las ciencias de la salud. $4^{\text {a }}$ ed. México: Limusa. 755 p.

3. Chigerwe M, Middleton JR, Williams F, Tyler JW, Kreeger JM. 2007 Atypical coccidiosis in South American camelids. J Vet Diagn Invest 19: 122125.

4. Fowler M. 1998. Medicine and surgery of South American camelids. $2^{\text {nd }}$ ed. Iowa, USA: Iowa State University Press. $531 \mathrm{p}$.

5. Guerrero C, Bazalar H, Leguía G. 1970. Prevalencia de coccidias (Protozoa: Eimeriidae) en alpacas (Lama pacos). Bol Extraord Lima Rev Fac Med Vet UNMSM 4: 84-90.

6. [INEI] Instituto Nacional de Estadística e Informática. 2007. Censos nacionales 2007, XI de población y VI de vivienda. Sistema de consulta de principales indicadores demográficos, sociales y Económicos. [Internet]. Disponi- 
ble en: http://censos.inei.gob.pe/Censos2007/IndDem/\#

7. [INEI] Instituto Nacional de Estadística e Informática. 2010. Sistema de información regional para la toma de decisiones. [Internet]. Disponible en: http:/ /webinei.inei.gob.pe:8080/SIRTOD/ inicio.html\#app $=8 \mathrm{~d} 5 \mathrm{c} \& \mathrm{~d} 4 \mathrm{a} 2$ selectedIndex $=0 \& \mathrm{~d} 9 \mathrm{ef}$-selectedIndex $=0$

8. Leguía G, Casas E. 1999. Enfermedades parasitarias de camélidos sudamericanos y atlas parasitológico de camélidos sudamericanos. Lima: Ed de Mar. 190 p.

9. Mamani P, Condemayta C, Calle $C$. 2009. Causas de mortalidad de alpacas en tres principales centros de producción ubicados en puna seca y húmeda del departamento de Puno. Red Vet 10 (8): 13p. [Internet]. Disponible en: http:// www.veterinaria.org/revistas/redvet/ n080809/080904.pdf

10. Martín C, Pinto C, Cid M. 2010. Camélidos sudamericanos: estado sanitario de sus crías. Rev Complutense Cienc Vet 4(1): 37-50.

11. Ocola J. 2008. Conozca los peligros y vulnerabilidades de los distritos de Crucero, Ajoyani, Corani, Macusani. [Internet]. Disponible en: http:// www.predes.org.pe/predes/images/ peligros_vulnerabilidades_carabaya.pdf

12. Palacios C, Tabacchi N, Chavera A, López U, Santillán A, Sandoval N, Pezo C, Perales R. 2004. Eimeriosis en crías de alpacas: estudio anátomo histopatológico. Rev Inv Vet Perú 15: 174-178. doi: 10.15381/rivep.v15i2.1602

13. Palacios C, Perales $R$, Chavera A, López T, Braga U, Moro M. 2006. Eimeria macusaniensis and Eimeria ivitaensis co-infection in fatal cases of diarrhea in young alpacas (Lama pacos) in Peru. Vet Rec 158: 344-345.

14. Radostits O, Gay C, Blood D, Hinchcliff K. 1999. Coccidiosis. En: Tratado de las enfermedades del ganado bovino, ovino, porcino, caprino y equino. $9^{a}$ ed. España: McGraw Hill. p 1538-1549.
15. Ramírez A, Enrique F, Pezo C. 1998. Diagnóstico y control de enfermedades en camélidos sudamericanos. Publ Téc Fac Med Vet San Marcos 34: 36-39.

16. Rodríguez A, Casas E, Luna L, Gavidia C, Zanabria V, Rosadio $R$. 2012. Eimeriosis en crías de alpacas: prevalencia y factores de riesgo. Rev Inv Vet Perú 23: 289-298. doi: 10.15381/ rivep.v23i3.911

17. Rojas M. 2004. Nosoparasitosis de los rumiantes domésticos peruanos. $2^{\mathrm{a}} \mathrm{ed}$. Lima: Martegraf. $146 \mathrm{p}$.

18. Romero M. 1992. Prevalencia y carga parasitaria de Eimeria spp en crías de alpacas. Tesis de Médico Veterinario. Univ Nacional Mayor de San Marcos. $28 \mathrm{p}$.

19. Rosadio R, Ameghino E. 1994. Coccidial infections in neonatal Peruvian alpacas. Vet Rec 135: 459-460.

20. Rosadio R, Londoñe P, Pérez D, Castillo H, Véliz A, Llanco L, Yaya K, Maturrano L. 2010. Eimeria macusaniensis associated lesions in neonate alpacas dying from enterotoxemia. Vet Parasitol 168: 116-120. doi: 10.1016/j.vetpar.2009.10.010

21. Rosadio R, Maturrano L, Pérez, D, Luna L. 2012. El complejo entérico neonatal en alpacas andinas. Rev Inv Vet Perú 23: 261-271. doi: 10.15381/ rivep.v23i3.908

22. [SENAMHI] Servicio Nacional de Meteorología e Hidrología. 2010. Estación Macusani, Tipo Convencional Metereológica. [Internet]. [Internet]. Disponible en: http://www.senamhi. gob.pe/include_mapas/ddat_esta_tipo. php? estaciones $=00077 \overline{7}$

23. Wolf D. 2010. Untersuchungen zur Seroprävalenz von zystenbildenden Kokzidien und zu Gastrointestinal parasitosen bei Neuweltkameliden in Peru. PhD Thesis. Universität Gießen. 154 p. [Internet]. Disponible en: http:// geb.uni-giessen.de/geb/volltexte/2011/ 8087/pdf/WolfDenis_2010_12_13_.pdf 